\title{
Efficient Energy Utilization in Cloud Fog Environment
}

\author{
Babur Hayat Malik ${ }^{1}$, Muhammad Nauman $\mathrm{Ali}^{2}$, Sheraz Yousaf ${ }^{3}$, Mudassar Mehmood $^{4}$, Hammad Saleem $^{5}$ \\ University of Lahore, Gujrat Campus
}

\begin{abstract}
Cloud computing provides various kind of services like storage and processing that can be accessed on-demand when required. Despite its countless benefits, it incorporates some issues too that limits the full adaption of cloud and enjoy its various benefits. Mainly the issue faced during the adaptability of cloud infrastructure is high latency and unawareness of location. To overcome these issues the concept of fog computing is introduced to reduce the load on the cloud and improve the allocation of resources. The fog provides the same services as the cloud. The main features of fog are; location awareness, low latency, and mobility. However, increasing the use of IoT devices, also increase the usage of Cloud. Fog environment. So, much usage of fog getting attention of researcher about energy consumption. In this paper, we try to solve the problem of energy consumption in terms of resources allocation by applying the load balancing algorithms and compare its result with the energy models.
\end{abstract}

Keywords-Energy efficiency; fog computing; cloud computing; load balancing; resources allocation

\section{INTRODUCTION}

The readers have to know about cloud computing, fog computing, and load balancing algorithm to understand the problem. So, cloud computing is a network or pool of physical and virtual resources over the internet, rather than personal hardware and soft- ware. We simply say that cloud is a delivery of computing services such as network, storage, database, and software over the internet. Need to pay for access the cloud services for using purpose. The architecture of cloud computing is -referred to its components and sub components. Fog architecture is described as in Fig. 1.

It can describe in four layers. First one is Front-end, it consists of the client-side devices. The second one is the back end, it consists of the servers that providing the services mean where the data is stored. It contains the cloud resource that demanded by the user. The third one the median between client and server it consists of software, web browser or the virtual session. It can be also called the middle-ware. And the fourth one is consists of the cloud-based delivery and networks. There are different cloud base deliveries Software as a Service (SaaS), Development as a Service (DaaS), Data as a Service (DaaS) and Platform as a service (PaaS). In SaaS, the client did not need to install any specific software required to connect to the server. Software service is already installed on the server side. Development as a service (Daas) is consisting of development tools. Data as a Service (Daas) is also considered as the subset of software in which cloud data is accessed by using APIs. Platform as a Service is service provide by cloud computing and it provides the service like as Platform and database as a service.
Fog computing extends the concept of cloud computing that contributes a productive solution to manage the fastly growing smart devices by simply adding the data to the edge of the network. Fog computing is introduced to reduce the load on cloud computing and fog have some features which are location awareness, low latency, and response faster compare to cloud. Fog is placed at the edge of networks and provides the fast transfer of cloud service and its appropriate architecture divided it in three-layer. The bottom most layer is consist of a client device that needed the service to utilize. It consists of mobile devices, clients, robots and any device that needed the cloud services and can connect to the fog computer. The Middle layer is consisting of the network that is used by server and clients to share services. The network layer consists of components like as router, switches, gateways and base station. And the third is the server layer mean cloud layer that provides the services.

Load balancing algorithms that can be used to intelligently to balance the load of client access requests across servers pool. The load balancing has some functions it distributes client requests or network load efficiently across multiple servers, ensures high availability and reliability by sending requests only to servers that are online, and provides the flexibility to add or subtract servers as demand dictates. The main problem in the server now a days are load and its distribution. Some node is too busy to process the data and some are not utilizing their resource. In load balancing, technique data is the load of data divided between the server in proper manners that every server should utilize its maximum resource and avoid latency. And there many load balancer algorithm that manage the load in an efficient way that is the Routers and Switches that intercept the data to a target server and redirect the traffic or send it to servers that are available [4]. The load has dynamic nature mean it varies with time so it depends on the end user client request. So when some server is overloaded we use load balancing technique to utilize the unutilized server. There are several factors should be considered for the load balancing that is the total load, scalability of servers, throughput, a performance of the system, interaction between the servers, amount of work to be transferred and selection of best nodes. The load can be a network, virtual or CPU load and load balancing can also be done through in many ways like as dynamic or static and centralized and non-centralized. In the static technique, we have a record of the past load and we apply the technique on the basis of the previous record while in dynamic load balance we focus on the current data and processed on the current based. So, dynamic is better than the static. 


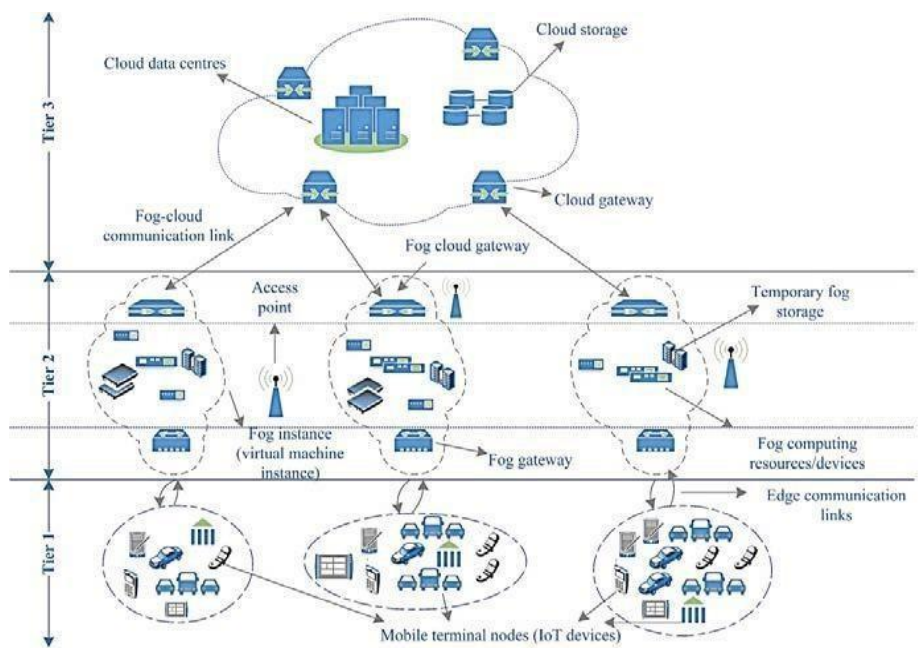

Fig. 1. Fog Architecture [3].

As we maintain the work burden on the cloud by adding the fog in the network but the as with the increasing rate of IoT devices we have to reduce the energy consumption on the fog computing. When the end user request for the resource from fog, during that time energy consumption can reduce by efficient allocation of re- source and here we are trying to solve the problem of resource utilization with respect to energy consumption. The energy consumption is based mostly on two things. The type of data to process and the requests and the energy consumption at the request level further is divided into three types. First is when the request is sent by the end user to the fog or received by fog. The second one is when the request is then processed by the fog and the third one is when fog requests the data from the cloud. Our focus is on the energy consumption of fog when it processes the request.

\section{A. Motivation}

Information technology plays an important role in computing. With the increase in the number of IoT devices, the request of resources increases on the cloud-fog [1]. So request response time, resources utilization and energy consumption have been critical issues nowadays. Fog computing act as a mid-layer between the end user and cloud that allocate the resources according to the request of the users which is closer to the end user to optimize the response time [2].On the other hand, load balancing is one of the important technique to allocate the resources and optimize the response time. And we want to improve resources allocation for reducing energy consumption by using load balance techniques.

\section{RELATED WORK}

The authors in [5], used matching game formulation to minimize the energy consumption in fog computing. In this formulation, matching game acts as a player between fog and devices.

According to authors the outcome of this model matching game that explained as a task of devices. The matching game scheme obtained the performance through effective coordination between fog and devices that connect with one another and choose their methodology dependent on the current conditions, the usage of the Fog content accessibility and the resource allocation management and putting away to get better execution. The authors say in this work proposed a matching game approach to deal with accomplishing lowenergy utilization in IOT and fog condition. The authors made utilization of cache technology and fog computing to enhance energy efficiency.

The authors in [6], introduced an investigation of fog computing as an answer for expanding the demand for IoT gadgets and focus on the energy utilization, quality of services. The authors contributing to investigating an issue of energy utilization of the fog computing with regards to IOT applications and proposed an energy-delay solution for the cloud-fog is an efficient computing model where resource, storage, and network infrastructure can be shared as a service through the internet. For this purpose the authors adopt the two optimization method which is BIP algorithm and improved the GA to solve the problem.

In [7], the authors propose a cloud and fog based condition for the management of energy. The goal of the author's energy cost minimization, carbon release minimization and to adjust to risky transmission supportable sources are destinations of this work. The authors developed a cloud-fog based structure for the viable management of the purchases request. Fog gives low idleness benefits as it is put near the end customer layer and can respond rapidly to customers' request. MGs are set near each fog which fulfills the power essentials by keeping up the supportability of structures. Response time and request changing time are enhanced using the BA. In this paper [8], the authors' cloud-fog based condition for effective energy management is proposed. Simulation in Cloud Analyst is performed to look at and analyze the execution of load balancing algorithms like Round Robin, Throttled, and Weighted Round Robin. Simulations results demonstrated that Throttled load balancing algorithm gives preferred reaction time over RR and WRR.

The authors in [9], consider the layer of architecture consists of edge, fog and cloud layers. At different layers, processing and forwarding data by devices power are consumed differently. The problem arises here how to identify which machine learning steps to be performed at which layer to minimize the energy consumption. The authors proposed fog based Ubicomp system consisting of edge, cloud and fog layer. An Ubicomp system which is consists of different units which is sensing, communication, processing, and storage. The authors present fog based architecture of Ubicomp system and analyze the energy consumption on the edge, fog and cloud devices by complete the system. Ubicomp has different devices which are sensing, processing devices and relay devices. The relay device forwards the data within the same layer and other layers in the network. The sensing devices can collect the data on edge devices from different senor and total data transmitted by a sensor for Ubicomp application. The Ubicomp applications also divide machine learning steps into data processing step and machine learning steps for figuring the results through processing devices. 
The authors in [10], concentrated on a cloud-fog based model is proposed for resource management in SG. The objective of this study is the efficient utilization of re-sources. The authors inspected the advantages and opportunities of cloud-fog to help resource management in the SG. In addition, a model is proposed to interface these spaces. The authors play out the assessment regarding processing time, reaction time and cost minimization. Later on, the authors intend to improve the usage of cloud-fog based SG, which incorporate more services and features. As the cloud and fog advance quickly engaged with SG to build productivity, there is a requirement for devices to analyze and study the advantage of the innovation. Summary of related work is described in Table I [10]:

In [11], the authors talked about resources allotment in the cloud-fog condition. The proposed system IST effectively assigns the resources to DCs and satisfies the DS request. The authors focus is around the allotment of VMs to DCs. The effectiveness of IST is figured by looking at the response time and Cost. It very well may be seen from the finished up results that the expense of the fog and cluster is nearly the equivalent. The response time of IST is same as the response time of the RR algorithm. Minimal effort and response time show the unwavering quality of this framework. Later on, the authors to crossover RR and IST process the execution of the present situation and furthermore in the wake of rolling out improvements in the present as well.

In [12], the authors plan to think about different energy effective procedures for the cloud which utilizes resources allotment and solidification to accomplish the equivalent. Further, the authors attempt to change the current procedure by including an additional parameter. The fundamental point of the author's works creates viable approaches and algorithms for virtualized data farms. Furthermore, attempt to reduce energy utilization with boosting the resource usage. The authors propose to include an additional parameter viz. time alongside cost, which may bring about boosting resources usage and limiting the number of running servers. The proposed thought is yet to be executed and tried in different constant or reenactment condition before its wide acknowledgment.

In [13], the authors focused issues of migrating virtual machines (VMs) among various physical hosts is crucial for resource use and carbon dioxide (CO2) minimization in cloud data centers. The proposed method CPU Utilization Variance (CUV) depends on choosing the best VMs from over utilized servers and moving them into different servers to spare the used resources and not to damage the SLA built up between clients and cloud service provider. Server link is a methodology used to reduce the total number of utilizing servers that an organization requires. Static VM combination techniques are not recommended to implement the experiments, in this way unique consolidation strategies should be utilized to enhance the resources use. In this paper, the proposed procedure is executed on a large scale server farm made out of 800 physical hosts. The results obtained by CloudSim apparatus are as far as energy utilization in $\mathrm{KW} /$ hour, execution in Million Instructions for every Second (MIPS), a number of VM relocations and SLAV. The recreation results demonstrate that CUV algorithm has a number of VM allocations and energy utilization superior to in future, the authors intend to build up the proposed algorithm to get the finest results of performance and SALV nor- mal results. The authors implement all investigations in real cloud organization using nearly open- source apparatuses like OpenStack which these days have a critical use in distributed computing situations.

In [14], the authors discuss the load management algorithms that are often used in cloud computing. After that, the authors perform an experiment by using one load balance technique on a VM in cloud computing. The method is tested in a cloud computing condition. It has two options to test the first option is to use real tests like Amazon EC2, and the second is to use a simulation tool to simulate the cloud environment. In this paper, it can be seen that load balancing is an essential viewpoint on the execution of existing distributed computing. Since a load of the demand from clients is specifically and moving. What's more, after that from this examination, the authors presume that the throttled algorithm utilized in load balancer in cloud computing recreations utilizing Cloud Analyst is the typical response rate is still inside the ordinary range among user-base 1 and the other. Be that as it may, in regards to the base normal and greatest response.

The world is distributed in regions shown in Fig. 2, and the every region has its own id given in Table II [14]. Paper Organization: The remaining part of this paper is divided in a category as: In Section 3, literatures has been discussed. Section 4 discussed the load balancing algorithm, Section 5 discussed the proposed approach, Section 6 discussed the experiment, evaluation setup and Section 7 discussed conclusion.

TABLE I. SUMMARY OF RELATED WORK

\begin{tabular}{|c|c|c|c|c|c|}
\hline \multirow[b]{2}{*}{ Authors } & \multirow{2}{*}{$\begin{array}{l}\text { Proposed } \\
\text { Algorithm }\end{array}$} & \multirow[b]{2}{*}{ Infrastructure } & \multirow{2}{*}{$\begin{array}{l}\text { Evaluation } \\
\text { Process }\end{array}$} & \multicolumn{2}{|c|}{ Method Proposed for } \\
\hline & & & & \begin{tabular}{|l|} 
Energy \\
Efficiency
\end{tabular} & $\begin{array}{l}\text { Resources } \\
\text { Allocation }\end{array}$ \\
\hline Assila et al.[5] & Matching Game & Fog & Cloud Analyst & Yes & Yes \\
\hline Zafar et al.[7] & BA & Cloud-Fog & Cloud Analyst & Yes & Yes \\
\hline Naeem et al.[8] & RR,WRR,Throttled & Cloud-Fog & Cloud Analyst & Yes & No \\
\hline Saraswat et al.[9] & ML & Cloud-Fog & Mathematical Analysis & Yes & No \\
\hline Sharif et al.[11] & IST & Cloud-Fog & Cloud Sim & No & Yes \\
\hline Patel et al.[12] & MaxUtil & Cloud & Theoretical Analysis & Yes & Yes \\
\hline
\end{tabular}


TABLE II. REGION DISTRIBUTION

\begin{tabular}{|l|l|}
\hline Region & Region Id \\
\hline North America & 0 \\
\hline South America & 1 \\
\hline Europe & 2 \\
\hline Asia & 3 \\
\hline Africa & 4 \\
\hline Oceans & 5 \\
\hline
\end{tabular}

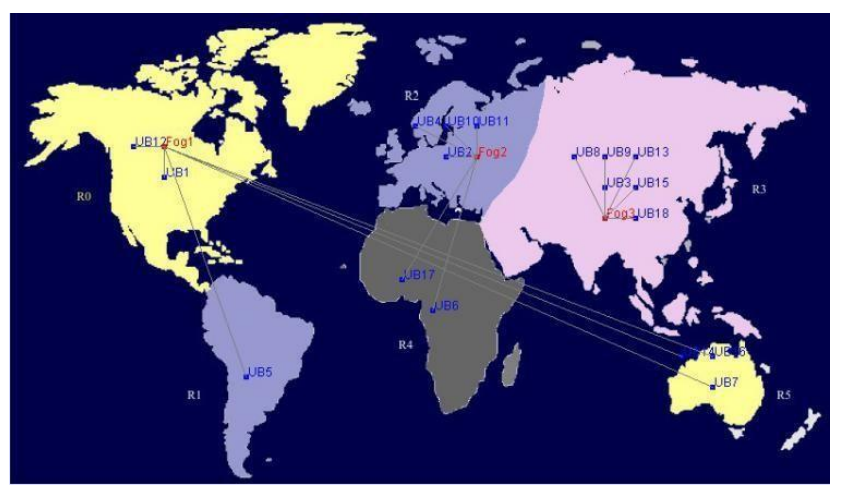

Fig. 2. Regions

\section{LOAD BALANCING ALGORITHMS}

Load balancing algorithms have an important role in the cloud fog environment. These algorithms are used to distribute the resource Received by the data center efficiently. In this paper, three load balancing algorithm are considered which are described are as follows:

1) Round Robin (RR): Round Robin is a time-sharing technique. It stores the entry of the user request with respect to arrival time in the table. It equally shares the time among the virtual machine. That makes the balance between the virtual machines.

2) Throttle (TT): Throttle working is different from the round robin. It checks the type of request and analyzes the virtual machines. And allocate the resource to that machine which has the capacity to full fill the task efficiently. This algorithm saves the state of every virtual machine in the table. The request received by the data center, it sends it to the load balancer. The load balancer checks the type of request and the virtual machines available to perform this task. If any machine is available then allocate the task to a virtual machine else it has to wait for the virtual machine.

3) Active VM: In the Active VM, the request received by the data center is stored in the queue. The data center checks the availability of the virtual machines. If the virtual machine available, then assigns the task to it and request removed from the queue. When the task is completed by the virtual machine, it will be available to perform another task.

We use these benchmarks because we want to compare our pro-posed algorithm performance with the previous algorithms results.

\section{PROPOSED APPROACH}

The energy consumption in fog is on three steps: 1) Receiving re- quest from end user, 2) Process the request, 3) Send request to cloud for resource but we focus on the second one which processing of the request. When request is received by the fog it check the VM's and send request to selected VM to process request. Our contribution here is to manage the sending of data for processing to VM's. We improve the Active VM load balance algorithm by adding threshold and sorting to manage this request, and consume less energy. This algorithm will check the VM distance, latency, processing speed and load then send request to the appropriate one. From this approach all virtual machines are utilized and burdens divided among the all machines which reduce the energy consumption. Before our algorithm the other only provide the list machine, the virtual machine was not managed in any order. So a machine on top can used more than one time as non-utilize machine will be not utilized until the first one complete its capacity. But in our Algorithm as a request is sent to the fog it receives and checks the status of the Virtual ma- chines and takes account of those machines that's lies between the fixed thresholds, then machines are sorted according to their status and the request is process with which have low load of data. As the algorithm checks the status of every VM on every request the sorting of the VM give the less consumed VM and the system utilize it. Which puts the nonutilize virtual machine on top. A request received by fog. Firstly, it checks the type of data or service requested. Then check the machine which relates to it, then check each virtual machine status and compare the latency ratio and available virtual machines, then send the request to the appropriate one which has less burden and consume less time. In this scenario, we can say that more time saved and the less energy consumed as shown in Fig. 3.

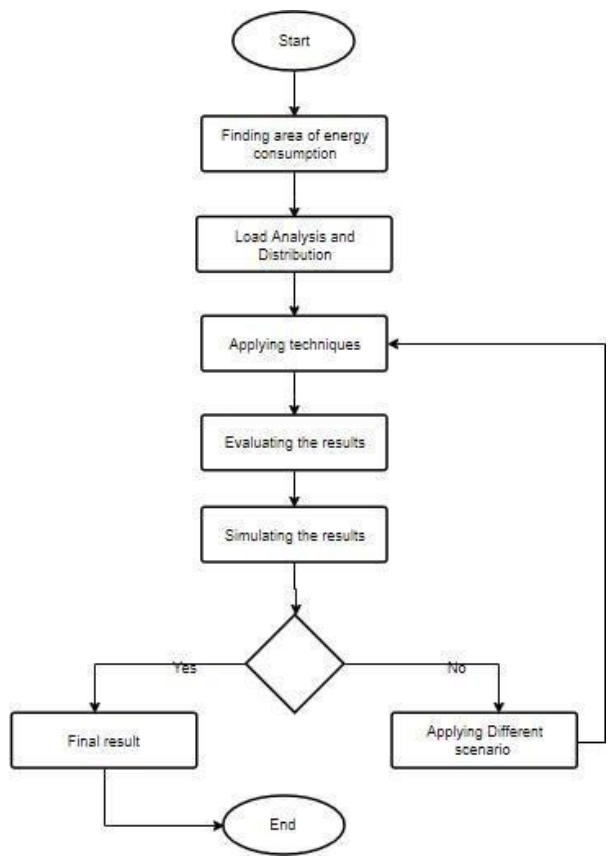

Fig. 3. Proposed Work. 


\section{A. Algorithm}

The main working of the IAVMis discussed above, the pseudocode of the algorithm describe the working of the main part of algorithm.

\begin{tabular}{l}
\hline Algorithm 1 IAVM \\
\hline 1: VMlist \\
2: Calculate $:$ Load ,VmCapicity \\
3: $V M$ List KirtualM achines \\
4: while(Vmlist $!=$ null $)$ : \\
5: if $V M$ list $<$ threshold then \\
6: $V M$ Stack virtualmachine \\
7: close; \\
8: $V M$ Stack.sort () \\
9: allocatedVm(VMStack.index) \\
\hline
\end{tabular}

In the first step, the load and capacity of every virtual machine are stored in the virtual machine list. Then a threshold sets to save those virtual machines into the stack that's been able to perform the given task, after that the virtual machines in the stack are sorted according to their capacity and availability. In the end, the resource is allocated to the virtual machine on the top and to other respectively.

\section{EVALUATION AND EXPERIMENTS}

We perform the experiments on the different simulator to test our assumptions. The example of one test that we performed is given below. We created six fog nodes on different location and they all are connected and each fog node contains the 6 virtual machine. We set the request sent to each server after five minutes. The size of the single request is 100 bytes and the request size varies after some time. Three algorithms are used to compared which are RR,

Throttle and Active VM: After saving the configuration we put the simulator for the simulation of result for 24 hours and save the results after simulation. Simulators evaluation is described in Table III.

The facts about the experiment are:

Energy $=$ No. of KWh $x$ Cost of one kWh.

TABLE III. SimULATORS EVALUATION

\begin{tabular}{|l|l|}
\hline Simulation Time & 24 Hours. \\
\hline Service Broker Policy & Closest Data Center \\
\hline
\end{tabular}

Cost model is described in Table IV [7]:

Data center hardware is mentioned below in Table V [12]:

Data center virtual machine specification is described below in Table VI [7]:

TABLE IV. COST MODEL

\begin{tabular}{|l|l|}
\hline VM cost & 0.1 per hour. \\
\hline Memory Usage Cost & 0.025 per second. \\
\hline Data Storage Cost & 0.05. \\
\hline Storage Cost & 0.05. \\
\hline
\end{tabular}

TABLE V. DATA CENTER HARDWARE CONFIGURATION

\begin{tabular}{|l|l|}
\hline Processors on physical machine & 2. \\
\hline Processing power & $75 \mathrm{MIPS}$. \\
\hline Storage devices & $75 \mathrm{~GB}$. \\
\hline Memory & $1 \mathrm{~GB}$. \\
\hline Internal bandwidth & $5000 \mathrm{Mbps}$. \\
\hline
\end{tabular}

TABLE VI. DATA CENTER VIRTUAL MACHINE SPECIFICATION

\begin{tabular}{|l|l|}
\hline RAM & $512 \mathrm{MB}$. \\
\hline Storage & $5 \mathrm{~GB}$. \\
\hline Architecture & $\mathrm{x} 86$. \\
\hline Operating system & Linux. \\
\hline Virtualization technique & Xen. \\
\hline Bandwidth & 5000 MBPS. \\
\hline
\end{tabular}

Firstly, we check the Service response time by performing the experiments on the Cloud Analyst. There are many tests that are performed and in each test, the data on every machine varies with time and simulate these results and to compare our result with previous studies. We compare our algorithm with the different load balancing algorithm.

1) Cost: We test the cost analysis by varying different parameter. The experimental results show that if the cost of the data centers the same for all then the total cost is the same for the improved algorithm and comparative algorithms. But if we make difference in the cost of the data center, then the cost of the RR and Throttle is increased but the total cost of the IAVM is decreased. So, it proves that our algorithm is cost efficient.

2) Response time and energy: After cost analysis we check the SRT (Service Response Time) and the NL (Network Latency). We perform our experiments on the cloud analyst. And for energy we perform experiment on ifogsim shown in Fig. 4 [11].

In both experiment we varies the parameter that effect the performance like as number of machine, data load and data center cost. The overall results shows that the new purposed algorithm performs best in most of scenarios. The result can be seen in Fig. 5 and 6 [11].

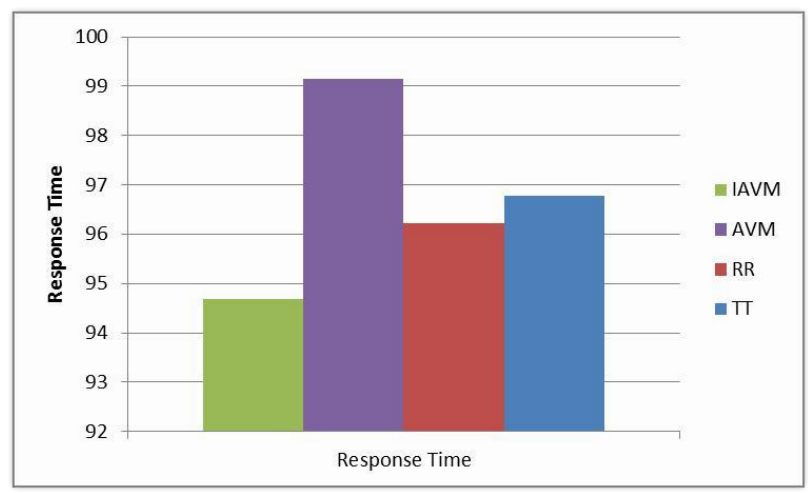

Fig. 4. Avg Response Time. 


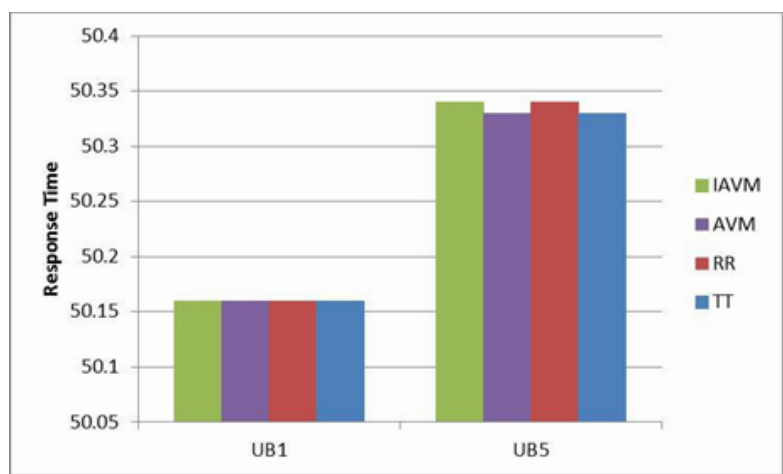

Fig. 5. Response time for user base 1 and 5 .

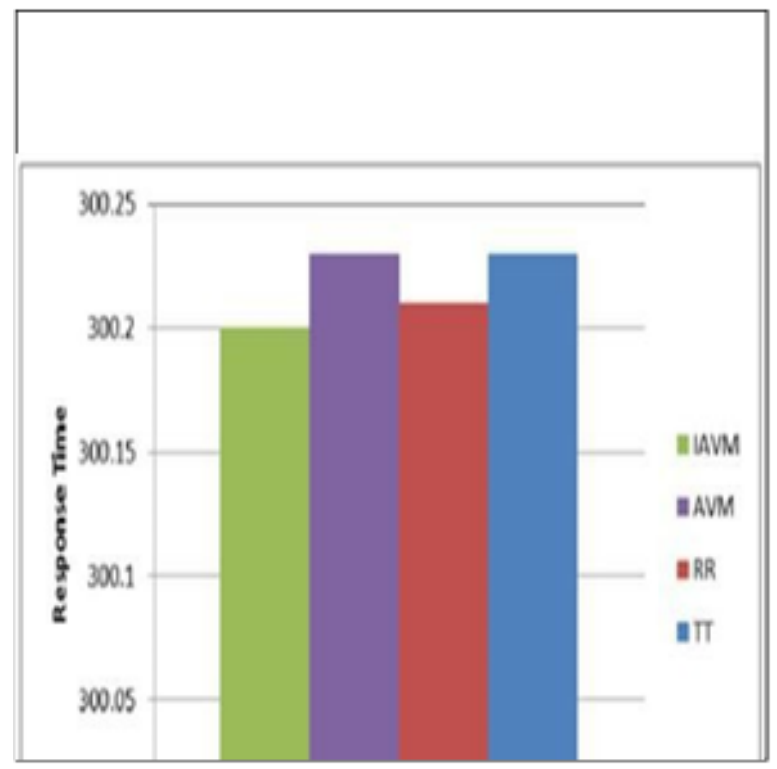

Fig. 6. Response Time for user base 3 .

As the load on the different fog node increases the response time of the fog node changed (Fig. 7 and 8). In different scenarios the response time variation shows the performance of the algorithm, and the results shows that in mostly scenarios the IAVM outperforms than others [4].

As the good response time improves the performance of system it as well as it minimizes the energy consumption in the fog environment. Because the load on each virtual machine efficiently distributed which increase the performance and reduce the energy consumption Fig. 9 [6].

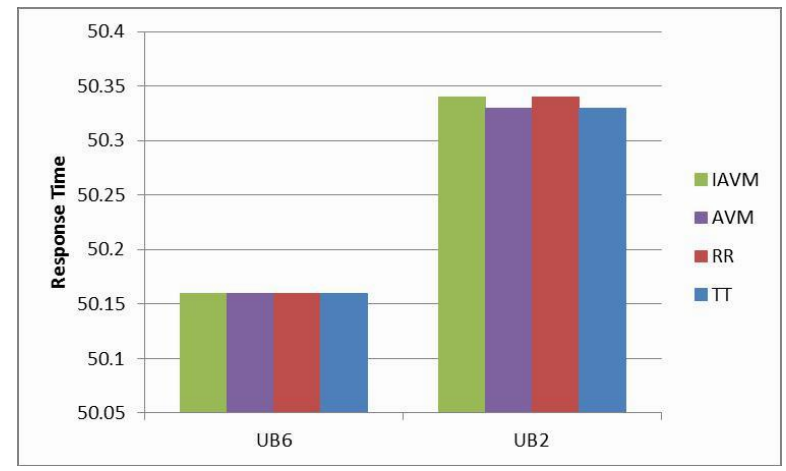

Fig. 7. Response Time for user base 2 and 6 .

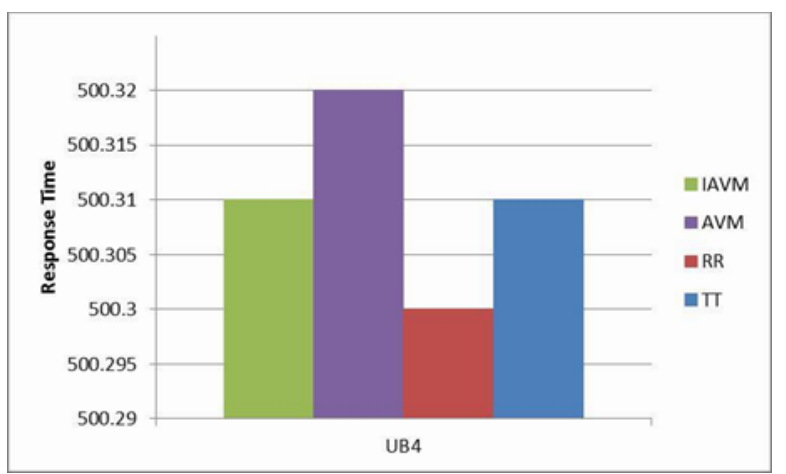

Fig. 8. Response Time for user base 4 .

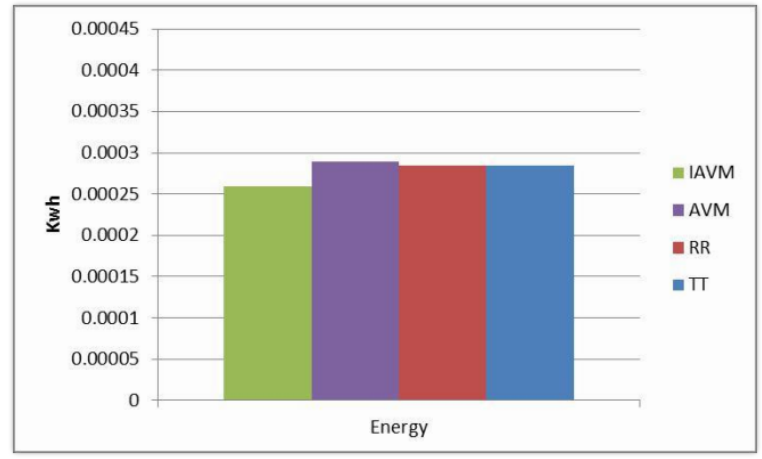

Fig. 9. Energy Consumption.

\section{CONCLUSION}

Due to the most advantages, the fog computing is becoming famous and the increase in the IoT devices increase the load on the fog computing. Due to the more the more processing, the power and energy consumption in cloud fog environment have become an issue due to the ecological and economic reasons. In this paper, we have presents an algorithm which distributes the resource in fog computing efficiently and minimizes the energy consumption in the cloud fog environment. The research results show that the purposed algorithm performs better than other. This paper will be helpful for efficient use of the resource in the cloud fog environment, which improve its performance in minimizing the energy consumption in Fog computing.

\section{REFERENCES}

[1] Okay, F. Y., Ozdemir, S. (2016, May). "A fog computing based smart grid model." In Networks, Computers and Communications (ISNCC), 2016 International Symposium on (pp. 1-6). IEEE

[2] Chiang, M., Zhang, T. (2016). "Fog and IoT: An overview of research opportunities." IEEE Internet of Things Journal, 3(6), 854-864

[3] Naas, M. I., Parvedy, P. R., Boukhobza, J., Lemarchand, L. (2017, May). iFogStor: "an IoT data placement strategy for foginfrastructure. In Fog and Edge Computing (ICFEC)", 2017 IEEE 1st International Conference on (pp. 97-104). IEEE

[4] Verma, M., Yadav, N. B. A. K. (2015). "An architecture for load balancing techniques for Fog computing environment." International Journal of Computer Science and Communication, 8(2),43-49.

[5] Assila, B., Kobbane, A., Walid, A., \& El Koutbi, M. (2018, May). "Achieving low-energy consumption in fog computing environment: A matching game approach.” In 2018 19thIEEE Mediterranean Electrotechnical Conference (MELECON) (pp. 213- 218). IEEE.

[6] Mebrek, A., Merghem-Boulahia, L., \& Esseghir, M. (2017, October). "Efficient green solution for a balanced energy consumption and delay in 
the IoT-Fog-Cloud computing." In Network Computing and Applications (NCA), 2017 IEEE 16th International Symposium on(pp. 1-4). IEEE.

[7] Zafar, F., Javaid, N., Hassan, K., Murtaza, S., Rehman, S., \& Rasheed.S. (2018, September). "Resource Allocation over Cloud-Fog Framework Using BA." In International Conference on Network-Based Information Systems (pp. 222-233). Springer, Cham.

[8] Naeem, M., Javaid, N., Zahid, M., Abbas, A., Rasheed, S., \& Rehman, S. (2018, September). "Cloud and Fog Based Smart Grid Environment for Efficient Energy Management." In International Conference on Intelligent Networking and Collaborative Systems (pp. 514-525). Springer, Cham.

[9] Saraswat, S., Gupta, H. P., \& Dutta, T. (2018, January). "Fog based energy efficient ubiquitous systems." In Communication Systems \& Networks (COMSNETS), 2018 10th International Conference on (pp. 439-442). IEEE.

[10] Zahoor, S., Javaid, N., Khan, A., Muhammad, F. J., Zahid, M., \& Guizani, M. (2018). "A cloud-fog-based smart grid model for efficient resource utilization." In 14th IEEE InternationalWireless
Communications and Mobile Computing Conference (IWCMC-2018).

[11] Sharif, M. U., Javaid, N., Ali, M. J., Gilani, W. A., Sadam, A., \& Ashraf, M. H. (2018, September). "Optimized Resource Allocation in FogCloud Environment Using Insert Select." In International Conference on Network-Based Information Systems (pp. 611-623). Springer, Cham.

[12] Patel, K., Patel, N., \& Patel, H. (2016, March). "Efficient Resource Allocation Strategy to Improve Energy Consumption in Cloud Data Centers." In Proceedings of the Second International Conference on Information and Communication Technology for Competitive Strategies (p. 76). ACM.

[13] Selim, G. E. I., El-Rashidy, M. A., El-Fishawy, N. A. (2016, February). "An efficient resource utilization technique for consolidation of virtual machines in cloud computing environments." In Radio Science Conference (NRSC), 2016 33rd National (pp. 316-324).IEEE.

[14] Ramadhan, G., Purboyo, T. W., Latuconsina, R. (2018). "Experi- mental Model for Load Balancing in Cloud Computing Using Throttled Algorithm." International Journal of Applied Engineering Research, 13(2), 1139-1143. 